\title{
Exploring myocardial fibrosis in severe aortic stenosis: echo, CMR and histology data from FIB-AS study
}

\author{
Giedrè Balčiūnaitė ${ }^{1}\left[\right.$ ] Justinas Besusparis ${ }^{1} \cdot$ Darius Palionis $^{1} \cdot$ Edvardas Žurauskas $^{1}$ - Viktor Skorniakov ${ }^{1}$. \\ Vilius Janušauskas ${ }^{1}$ - Aleksejus Zorinas ${ }^{1} \cdot$ Tomas Zaremba $^{1,2}$. Nomeda Valevičienè ${ }^{1}$. Pranas Šerpytis ${ }^{1}$. \\ Audrius Aidietis $^{1} \cdot$ Kęstutis Ručinskas ${ }^{1} \cdot$ Peter Sogaard $^{1,2} \cdot$ Sigita Glaveckaitè $^{1}$
}

Received: 8 December 2021 / Accepted: 25 January 2022 / Published online: 3 March 2022

(c) The Author(s), under exclusive licence to Springer Nature B.V. 2022

\begin{abstract}
Myocardial fibrosis in aortic stenosis is associated with worse survival following aortic valve replacement. We assessed myocardial fibrosis in severe AS patients, integrating echocardiographic, cardiovascular magnetic resonance (CMR) and histological data. A total of 83 severe AS patients (age $66.4 \pm 8.3,42 \%$ male) who were scheduled for surgical AVR underwent CMR with late gadolinium enhancement and T1 mapping and global longitudinal strain analysis. Collagen volume fraction was measured in myocardial biopsies (71) that were sampled at the time of AVR. Results. CVF correlated with imaging and serum biomarkers of LV systolic dysfunction and left side chamber enlargement and was higher in the sub-endocardium compared with midmyocardium $(p<0.001)$. CVF median values were higher in LGE-positive versus LGE-negative patients [28.7\% (19-33) vs 20.7\% (15-30), respectively, $p=0.040]$. GLS was associated with invasively (CVF; $\mathrm{r}=-0.303, p=0.013$ ) and non-invasively (native $\mathrm{T} 1 ; \mathrm{r}=-0.321, p<0.05$ ) measured myocardial fibrosis. GLS and native T1 correlated with parameters of adverse LV remodelling, systolic and diastolic dysfunction and serum biomarkers of heart failure and myocardial injury. Conclusion. Our data highlight the role of myocardial fibrosis in adverse cardiac remodelling in AS. GLS has potential as a surrogate marker of myocardial fibrosis, and high native T1 and low GLS values differentiated patients with more advanced cardiac remodelling.
\end{abstract}

Keywords Aortic stenosis · Myocardial fibrosis · Cardiovascular magnetic resonance $\cdot$ T1 mapping $\cdot$ Global longitudinal strain

$\begin{array}{ll}\text { Abbreviations } \\ \text { 6MWT } & \text { 6-Minute walking test } \\ \text { AS } & \text { Aortic stenosis } \\ \text { AV } & \text { Aortic valve } \\ \text { AVR } & \text { Aortic valve replacement } \\ \text { BNP } & \text { Brain natriuretic peptide } \\ \text { CAD } & \text { Coronary artery disease } \\ \text { CMR } & \text { Cardiovascular magnetic resonance } \\ \text { ECG } & \text { Electrocardiography } \\ \text { ECV } & \text { Extracellular volume } \\ \text { GLS } & \text { Global longitudinal strain } \\ \text { Hs-Tn-I } & \text { High-sensitivity troponin I }\end{array}$

Giedrẻ Balčiūnaitė

dr.giedre.balciunaite@gmail.com

1 Present Address: Vilnius University: Vilniaus Universitetas, Vilnius, Lithuania

2 Aalborg University Hospital, Clinical Institute of Aalborg University, Hobrovej 18-22, 9100 Aalborg, Denmark

$\begin{array}{ll}\text { LA } & \text { Left atrium } \\ \text { LGE } & \text { Late gadolinium enhancement } \\ \text { LV } & \text { Left ventricle } \\ \text { LVEF } & \text { Left ventricular ejection fraction } \\ \text { MLHFQ } & \begin{array}{l}\text { Minnesota Living With Heart Failure } \\ \text { Questionnaire }\end{array} \\ \text { NYHA } & \text { New York Heart Association } \\ \text { STE } & \text { Speckle tracking echocardiography }\end{array}$

\section{Introduction}

Myocardial fibrosis is fundamental in the pathogenesis of heart failure in the spectrum of cardiovascular diseases [1]. It is associated with the disruption of normal myocardial structure by excessive deposition of the extracellular matrix and creates a mechanistic base for adverse cardiac remodelling [2]. Myocardial fibrosis in aortic stenosis (AS) patients 
has been linked to impaired left ventricular (LV) function and adverse clinical outcomes [3].

Changes in cellular and extracellular matrix architecture, triggered by the greater afterload and wall stress in AS, increases tissue stiffness and impairs contraction $[4,5]$. This complex interplay between components of cardiac remodelling can be evaluated by histological analysis of myocardial biopsy samples or the use of advanced imaging techniques with ability of tissue characterization.

Cardiovascular magnetic resonance (CMR), strengthened by the development of $\mathrm{T} 1$ mapping, provides a non-invasive and global estimation of myocardial fibrosis. Two distinct types of myocardial fibrosis can be depicted by CMR: the late gadolinium enhancement (LGE) technique quantifies focal fibrosis [6,7], and diffuse interstitial expansion can be measured by T1 mapping [8]. Multicentre trials and meta-analyses have shown that the presence and extent of LGE are predictors of worse survival following aortic valve replacement (AVR), indicating advanced myocardial injury $[9,10]$. Focal myocardial fibrosis is also irreversible following AVR [11, 12], effecting incomplete recovery of LV function and worse post-operative clinical outcomes, suggesting delayed timing of aortic valve intervention in some patients.

Several studies in AS patients have reported that native $\mathrm{T} 1$ and extracellular volume (ECV) values correlate with the degree of diffuse myocardial fibrosis, predict cardiovascular events and mortality [13-15] and are reversible with afterload relief [16], demonstrating potential as an early marker of adverse remodelling.

As a possible surrogate marker of myocardial fibrosis, LV myocardial global longitudinal strain (GLS), as assessed by speckle tracking echocardiography (STE), has been shown to be an independent predictor of adverse events in patients with severe AS, both with preserved and impaired LV systolic function [17].

Thus, novel diagnostic strategies and more accurate evaluations of the disease severity and consequences of AS are needed in assessing subclinical myocardial dysfunction to further risk-stratify severe AS patients. There are limited studies on myocardial fibrosis that have integrated multimodality imaging and sufficient histological analyses in severe AS. The optimal T1 image analysis strategy remains debated, requiring further validation. Our prospective study aims to: (i) noninvasively assess markers of myocardial fibrosis and validate them against histological data in patients who are undergoing surgical AVR and (ii) identify early imaging biomarkers of adverse LV remodelling in severe AS patients.

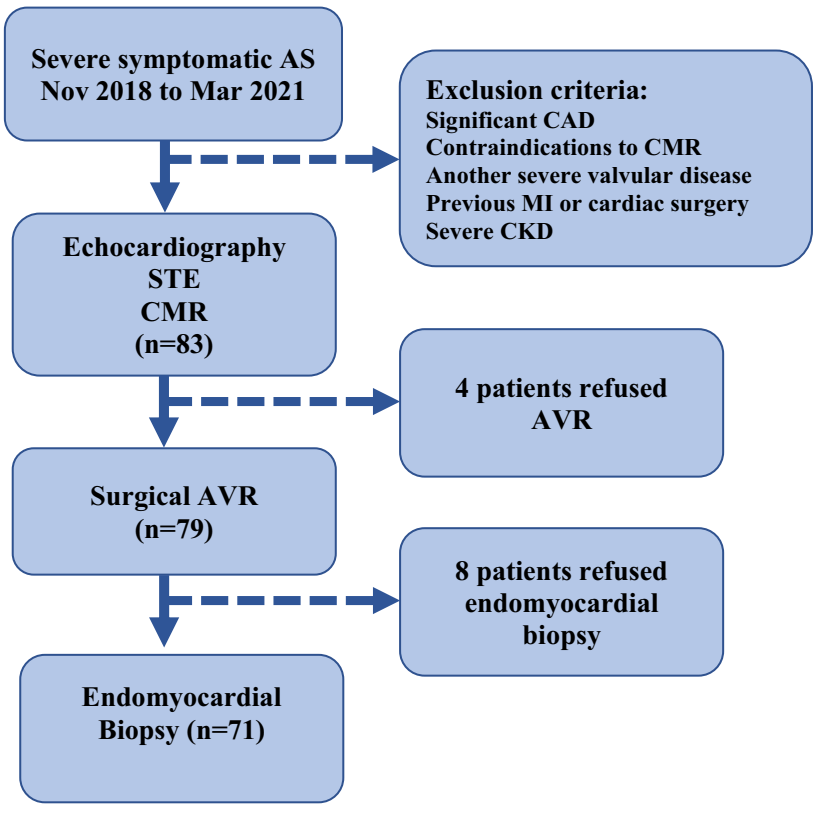

Fig. 1 FIB-AS study flow chart

\section{Methods}

\section{Study population and protocol}

In this prospective observational study at Vilnius University Hospital between November 2018 and December 2020, patients with severe symptomatic AS that were scheduled for AVR according to current treatment recommendations [18] were recruited. The study was approved by the Biomedical Research Ethics Committee of the Vilnius Region (Approval Number: 158200-18/9-1014-558) and was performed as part of the FIB-AS study (NCT03585933). This study conformed to the principles of the Helsinki Declaration, and all subjects gave written consent to participate.

Patients were recruited prior to a pre-operative assessment and underwent a clinical assessment, comprising a clinical history, the Minnesota Living with Heart Failure Questionnaire (MLHFQ), the 6-min walking test (6MWT), blood sampling [for haematocrit, renal function, brain natriuretic peptide (BNP) and high sensitivity troponin I (Hs-Tn-I)], a transthoracic echocardiogram, and CMR. The inclusion criteria were patients who were undergoing AVR for severe AS [defined as aortic valve area (AVA) $\leq 1 \mathrm{~cm}^{2}$ or AVA index $\leq 0.6 \mathrm{~cm}^{2} / \mathrm{m}^{2}$, as determined by ultrasonography], age $>18$ years, ability to undergo a CMR scan, and consent to the study protocol. The exclusion criteria were 
significant coronary artery disease (CAD) ( $>50 \%$ lesion), history of myocardial infarction, severe valve disease other than AS, estimated glomerular filtration rate $<30 \mathrm{~mL} /$ $\min / 1.73 \mathrm{~m}^{2}$, CMR-incompatible devices, persistent atrial tachyarrhythmias, and previous cardiac surgery (Fig. 1). The study data were collected and stored in a dedicated online database, REDCap (Research Electronic Data Capture) [19].

\section{Cardiac imaging}

\section{Echocardiography}

Transthoracic 2D echocardiography was performed using a commercially available Vivid ultrasound system (S70, E9 or E95) (GE Healthcare, Horten, Norway), and the data were stored on a dedicated workstation for subsequent off-line analysis. AS severity and LV systolic and diastolic function were evaluated per the echocardiographic guidelines [20, 21]. AVA was calculated using the continuity equation.

\section{The 2D speckle tracking echocardiography (STE)}

From the 2D grey-scale images of the apical 2-, 3- and 4-chamber views, LV global longitudinal strain (GLS) was measured and processed off-line using commercially available software (EchoPac 112.0.1, GE Medical Systems, Horten, Norway) [22]. The frame rate was adjusted to 50 to $80 \mathrm{frames} / \mathrm{s}$. End-systole was defined, based on the closure click on the spectral tracing of the pulsed-wave Doppler of AV flow. GLS was acquired using the average regional strain curves (17-segment model for 2D STE). Segments with poor quality tracking or aberrant curves (despite manual adjustment) were removed from analysis. Due to missing data or poor image quality, STE analysis was completed for 77 of 83 patients.

\section{CMR Protocol}

CMR scans were obtained using standard protocols on a 1.5 T Siemens Aera scanner with surface coils and retrospective electrocardiography (ECG) triggering. LV end-systolic and end-diastolic diameters and maximum wall thickness were traced and recorded from the short-axis and long-axis views of the standard ECG-gated steady-state-free precession cine sequence. $\mathrm{LV}$ volumes, mass and ejection fraction were measured using commercial software (suiteHEART®) from a stack of sequential 8-mm short-axis slices (0-2-mm gap) from the atrio-ventricular ring to the apex. Measurements were indexed to body surface area in $\mathrm{m}^{2}$ (using the DuBois formula).

\section{LGE Imaging}

To detect late gadolinium enhancement, images were acquired 10-15 min after intravenous administration of gadobutrol $(0.2 \mathrm{mmol} / \mathrm{kg}$ ) (Gadovist, Bayer AG, Germany) using a breath-hold segmented inversion recovery fast-gradient echo sequence in the short-axis and long-axis planes of the $\mathrm{LV}$, with an 8-mm slice thickness and $0 \%$ distance factor. The region of myocardial fibrosis was defined as the sum of pixels with a signal intensity above 5 standard deviations of the normal remote myocardium in each short-axis slice. The presence of LGE was determined qualitatively by two independent readers who were blinded to the clinical data.

\section{T1 Mapping}

Myocardial fibrosis was assessed using native and post contrast T1 mapping at a mid-ventricular short-axis section, acquired using a modified Look-Locker inversionrecovery (MOLLI) sequence with motion correction (the '3-3-5'standard protocol) before and 15 min after contrast administration [23]. Scanner generated T1 maps were processed off-line using commercially available software (suiteHEART by NeoSoft). The region of interest was manually traced on short-axis, native and post-contrast T1 maps in the septum at the mid-ventricular level. All T1-related measures were traced in the middle third of myocardium to avoid partial volume effects. Segments containing LGE were excluded from the T1 mapping analysis [24]. To measure the T1 value

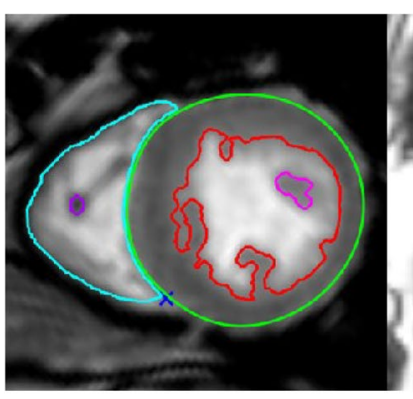

Short axis cine

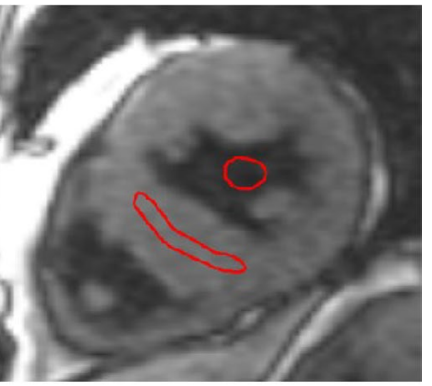

Native T1 map

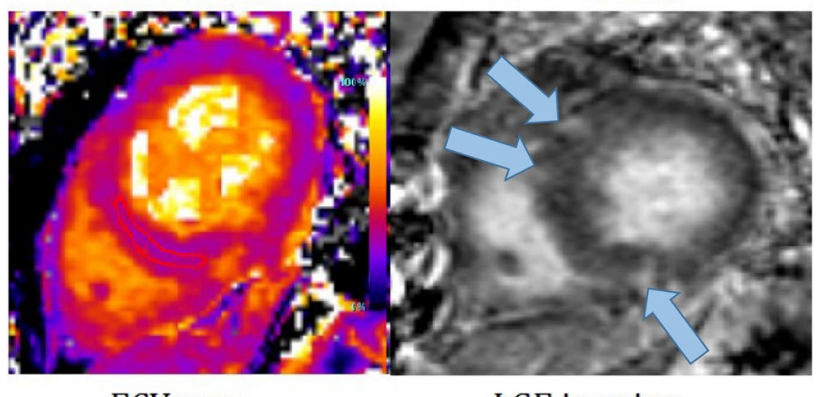

ECV map

Fig. 2 Multiparametric CMR assessment protocol 
of blood, circular regions of interest were positioned in the LV cavity, avoiding papillary muscle (Fig. 2). Native T1, $\mathrm{ECV} \%$, and indexed ECV values were then calculated. The ECV of the myocardium was calculated as follows: $\mathrm{ECV} \%=(\Delta \mathrm{R} 1 \mathrm{~m} / \Delta \mathrm{R} 1 \mathrm{~b}) \times(1$-haematocrit level $) \times 100$, where $\mathrm{R} 1$ is $1 / \mathrm{T} 1, \mathrm{R} 1 \mathrm{~m}$ is $\mathrm{R} 1$ in the myocardium, $\mathrm{R} 1 \mathrm{~b}$ is $\mathrm{R} 1$ in the blood and $\Delta \mathrm{R} 1$ is the change in relaxation [25]. Hematocrit was drawn on the day of CMR scanning. Due to incomplete datasets, $\mathrm{T} 1$ mapping parameters were measured in 67 of 83 patients.

\section{Histological analysis}

At the time of surgical AVR, biopsy specimens were obtained under direct vision by the surgical team using a surgical scalpel from the basal anteroseptum just after removal of the diseased AV. One intraoperative myocardial biopsy sample (mean area $22.5 \pm 12 \mathrm{~mm}^{2}$ ) was taken from each patient. All myocardial tissue samples were fixed in $10 \%$ neutral buffered formalin and embedded in paraffin. Sections (3- $\mu$ m-thick) were sliced on a Leica RM2145 microtome and stained with haematoxylin and eosin and Masson 's trichrome. Digital images were captured by on an Aperio Scan-Scope XT Slide Scanner (Aperio Technologies, Vista, CA, USA) under $20 \times$ objective magnification $(0.5 \mu \mathrm{m}$ resolution). Histologists who were blinded to the clinical and CMR data examined all biopsy specimens.

The fraction of myocardial volume that was occupied by collagen tissue (collagen volume fraction, CVF) was determined by quantitative morphometry on an automated image analysis system (HALO ${ }^{\mathrm{TM}}$ ). The area of myocardial fibrosis was calculated using the HALO ${ }^{\text {TM }}$ Area Quantification v2.1.11 algorithm (IndicaLabs, NM, USA) [26]. The subendocardial layer was defined as $1 \mathrm{~mm}$ from the endocardial surface, whereas the rest of the tissue sample was defined as the midmyocardial layer.

\section{Statistical analysis}

Variables were presented as mean \pm standard deviation or median and interquartile ranges. Categorical variables were expressed as frequencies and percentages and were compared by v2 test. Unpaired student's t-test and Mann-Whitney $U$ test were used to compare two groups of continuous variables. Pearson's and Spearman's correlation coefficients were calculated to assess the relationships between continuous variables.

Intra- and inter-observer variation was analysed by Bland-Altman method and calculation of the correlation coefficient. The statistical analysis was performed in $\mathrm{R}$ (version 4.1.2). Differences were considered statistically significant provided a 2 -sided $p$ value $<0.05$ [27].

\section{Results}

A total of 83 patients were included (age $66.4 \pm 8.3$ years, $58 \%$ female, AVA index $0.44 \pm 0.1 \mathrm{~cm}^{2} / \mathrm{m}^{2}$, peak AV velocity $4.8 \pm 0.6 \mathrm{~m} / \mathrm{s}$, mean gradient $57.8 \pm 16 \mathrm{~mm} \mathrm{Hg}$ ). The main reasons for non-eligibility were significant $C A D$, renal dysfunction and other valvular abnormalities. The mean LV ejection fraction (LVEF) was $66.8 \pm 13 \%$, with $11 \%$ of patients having reduced LVEF $(<50 \%)$. Overall, patients had low surgical risk, with STS-PROM and EuroScore $<4 \%$ (1.9\% and $1.5 \%$, respectively). Patients with congenital AS were more likely to be younger $(p<0.001)$, were at lower surgical risk $(p=0.004)$, and had better renal function $(p=0.002)$. Of the 83 enrolees, 79 underwent surgical AVR and 4 postponed surgery due to Covid-19. The patients' clinical and imaging characteristics are summarised in Tables 1 and 2.

\section{Myocardial fibrosis by histology}

Of 71 myocardial biopsies, 2 were epicardial. The data of one patient was excluded from the analysis due to an incidental finding of toxoplasmic myocarditis. The median CVF was $15.1 \%$ (8.6-21). Patients with higher CVF had a greater prevalence of hypertension $(p=0.024)$ and dyslipidaemia $(p=0.036)$. Higher values of CVF were observed in LGEpositive versus LGE-negative patients-28.7\% (19-33) vs $20.7 \%$ (15-30), respectively $(p=0.040)$. No significant differences in median CVF value were noted between patients with and without CAD [17.2\% (10-23) vs $13.4 \%$ (9-19), respectively; $p=0.094$ ]. Segmental analysis of myocardial biopsies revealed more fibrosis in the subendocardial layer compared with a midmyocardial layer [21.1\% (12-29) vs $8 \%$ (5-12); $p<0.001$; Fig. 3).

\section{Myocardial fibrosis by CMR}

The median delay between CMR and surgery was 53.3 days (17-78). Mean native T1 was $959.7 \pm 34 \mathrm{~ms}$ (range: 897-1044 ms), and the mean ECV was $22.7 \pm 3.6 \%$ (range: 15.7-34.4\%). No significant difference in mean native T1 and ECV values was observed between men and women $(962 \pm 29 \mathrm{~ms}$ vs $957 \pm 37 \mathrm{~ms}, p=0.391$ and $22.9 \pm 3 \%$ vs. $22.6 \pm 4 \%, p=0.821$, respectively).

To compare native $\mathrm{T} 1$ with clinical and structural parameters, we divided variables (above and below the median: $957 \mathrm{~ms}$, Table 3). Patients with elevated native T1 had lower systolic blood pressure $(p=0.006)$, higher QRS voltage on the ECG $(p=0.036)$, greater systolic $(p=0.009)$ and diastolic LV dimensions $(p=0.049)$ and higher LV mass index $(p=0.021)$. Among those with elevated native T1, a higher 
Table 1 Clinical characteristics of the study population stratified by the presence of focal fibrosis

\begin{tabular}{|c|c|c|c|c|}
\hline Variable & All patients $(n=83)$ & $\begin{array}{l}\text { LGE }(+) \\
\text { patients } \\
(n=61)\end{array}$ & LGE (-) patients $(n=22)$ & $P$-value \\
\hline Age, yrs & $66.4 \pm 8.3$ & $65.8 \pm 8.3$ & $68.3 \pm 8.3$ & 0.235 \\
\hline Male gender & $35(42 \%)$ & $29(48 \%)$ & $6(27 \%)$ & 0.162 \\
\hline BMI, $\mathrm{kg} / \mathrm{m}^{2}$ & $30 \pm 5.8$ & $30.4 \pm 5.6$ & $28.7 \pm 6$ & 0.245 \\
\hline BSA, $\mathrm{m}^{2}$ & $1.9 \pm 0.2$ & $2.0 \pm 0.2$ & $1.8 \pm 0.2$ & 0.011 \\
\hline Systolic BP, mmHg & $150 \pm 25$ & $148 \pm 25$ & $156 \pm 23$ & 0.223 \\
\hline Diastolic BP, mmHg & $85 \pm 11$ & $84 \pm 12$ & $85 \pm 11$ & 0.842 \\
\hline \multicolumn{5}{|l|}{ Comorbidities } \\
\hline Hypertension & $73(88 \%)$ & $55(90 \%)$ & $19(86 \%)$ & 0.732 \\
\hline Dyslipidemia & $66(80 \%)$ & $48(79 \%)$ & $19(86 \%)$ & 0.640 \\
\hline Unobstructive CAD & $39(47 \%)$ & $30(49 \%)$ & $9(41 \%)$ & 0.677 \\
\hline Diabetes mellitus & $14(17 \%)$ & $10(16 \%)$ & $5(22 \%)$ & 0.735 \\
\hline Atrial fibrillation & $6(7 \%)$ & $5(8 \%)$ & $1(5 \%)$ & 0.931 \\
\hline History of PCI & $1(1 \%)$ & $1(2 \%)$ & - & 1.000 \\
\hline \multicolumn{5}{|c|}{ Symptoms and functional status } \\
\hline Dyspnea & $61(74 \%)$ & $46(75 \%)$ & $15(68 \%)$ & 0.706 \\
\hline Chest pain & $41(49 \%)$ & $30(49 \%)$ & $11(50 \%)$ & 1.000 \\
\hline Syncope & $9(11 \%)$ & $9(15 \%)$ & - & 0.131 \\
\hline NYHA functional class & & & & $0.591^{*}$ \\
\hline I & $16(19 \%)$ & $11(18 \%)$ & $5(23 \%)$ & \\
\hline II & $24(29 \%)$ & $19(31 \%)$ & $5(23 \%)$ & \\
\hline III & $40(48 \%)$ & $28(46 \%)$ & $12(54 \%)$ & \\
\hline IV & $3(4 \%)$ & $3(5 \%)$ & - & \\
\hline $6 \mathrm{MWT}, \mathrm{m}$ & $357.6 \pm 105.6$ & $352 \pm 108$ & $372 \pm 101$ & 0.459 \\
\hline MLHFQ score & $35 \pm 20.4$ & $36 \pm 20$ & $31 \pm 22$ & 0.277 \\
\hline \multicolumn{5}{|l|}{ Drug history } \\
\hline ACE-I/ARB & $61(74 \%)$ & $43(71 \%)$ & $18(82 \%)$ & 0.453 \\
\hline Betablocker & $57(69 \%)$ & $42(69 \%)$ & $15(68 \%)$ & 1.000 \\
\hline Statin & $54(65 \%)$ & $40(66 \%)$ & $14(64 \%)$ & 1.000 \\
\hline Loop diuretic & $15(18 \%)$ & $11(18 \%)$ & $4(18 \%)$ & 1.000 \\
\hline Spironolactone & $22(27 \%)$ & $14(23 \%)$ & $8(36 \%)$ & 0.347 \\
\hline \multicolumn{5}{|l|}{ Risk scores } \\
\hline STS-PROM, \% & $1.9(1.2-2.3)$ & $1.6(1-2.2)$ & $1.75(1.4-2.4)$ & 0.415 \\
\hline EuroSCORE II, \% & $1.5(0.7-1.6)$ & $1(0.7-1.7)$ & $1.2(0.8-1.5)$ & 0.415 \\
\hline \multicolumn{5}{|l|}{ Surgery $(n=79)$} \\
\hline Tissue valve & $70(89 \%)$ & $55(90 \%)$ & $15(83 \%)$ & $\mathbf{0 . 0 3 7}$ \\
\hline Mechanical valve & $9(11 \%)$ & $6(10 \%)$ & $3(17 \%)$ & 0.927 \\
\hline Aortic intervention & $3(4 \%)$ & $1(2 \%)$ & $2(11 \%)$ & 0.348 \\
\hline \multicolumn{5}{|l|}{ Valve morphology } \\
\hline Tricuspid & $54(65 \%)$ & $41(67 \%)$ & $13(59 \%)$ & 0.671 \\
\hline Bicuspid & $28(34 \%)$ & $19(31 \%)$ & $9(41 \%)$ & 0.429 \\
\hline Unicuspid & $1(1 \%)$ & $1(2 \%)$ & - & 1.000 \\
\hline \multicolumn{5}{|l|}{ Blood tests } \\
\hline Creatinine $\mu \mathrm{mol} / 1$ & $76.2 \pm 16.3$ & $77 \pm 17$ & $73.9 \pm 16$ & 0.447 \\
\hline $\mathrm{eGFR}, \mathrm{ml} / \mathrm{min} / 1.73 \mathrm{~m}^{2}$ & $78.6(69-90)$ & 85 (69-90) & $86(69-90)$ & 0.996 \\
\hline Hs-Tn-I, pg/l & $10(5-19)$ & $13.5(6-29)$ & $5.3(5-9)$ & 0.003 \\
\hline $\mathrm{BNP}, \mathrm{pg} / \mathrm{l}$ & $122(65-340)$ & $167(77-511)$ & $74(43-145)$ & 0.004 \\
\hline \multicolumn{5}{|l|}{ ECG parameters } \\
\hline Heart rate, beats/min & $77 \pm 12.4$ & $78 \pm 12$ & $76 \pm 13$ & 0.519 \\
\hline S-L criteria (mm) & $30.8 \pm 10$ & $31.7 \pm 10$ & $28.4 \pm 10$ & 0.189 \\
\hline
\end{tabular}


Table 1 (continued)

\begin{tabular}{lllll}
\hline Variable & All patients $(n=83)$ & $\begin{array}{l}\text { LGE }(+) \\
\text { patients } \\
(n=61)\end{array}$ & LGE (-) patients $(n=22)$ & $P$-value \\
\hline QRS duration, ms & $96.8(88-102)$ & $94(88-102)$ & $92(85-101)$ & 0.449
\end{tabular}

The boldface values indicate statistical significance

Continuous variables are presented as mean \pm SD or median [interquartile range]. Categorical variables are expressed as $\mathrm{n}(\%)$

$6 M W T 6$ min walking test, $B M I$ Body mass index, $B N P$ Brain natriuretic peptide, $B P$ Blood pressure, $B S A$ Body surface area, $C A D$ Coronary artery disease, $E C G$ Electrocardiography, $L G E$ Late gadolinium enhancement, MLHFQ Minnesota living with heart failure questionnaire, NYHA New York Heart Association, PCI Percutaneous coronary intervention, $S$ - $L$ Sokolow Lyon voltage criterion, STS Society of Thoracic Surgeons' risk model score, EuroScoreII European System for Cardiac Operative Risk Evaluation II score, $A C E-I$ Angiotensin-converting-enzyme inhibitor, $A R B$ Angiotensin-receptor blocker, $h s-T n-I$ High sensitivity troponin I, $e G F R$ Estimated glomerular filtration rate

*- $P$-value for comparison among NYHA I and II vs. III and IV

proportion of patients had reduced GLS (18\% vs $6 \%$, respectively; $p=0.049$ ).

Focal fibrosis, measured by LGE, was common, affecting $74 \%$ of all patients ( $83 \%$ of men and $67 \%$ of women). Further, $92 \%$ of focal fibrosis was the non-infarct type (89\% mid-myocardial, $3 \%$ subepicardial). Despite having unobstructed coronary arteries $8 \%$ of patients had infarct-type focal fibrosis. The most common location of LGE was the right ventricular insertion point $(68 \%)$. LGE was also detected in the anterolateral (11\%), septal (8\%), posterolateral $(6 \%)$, inferior $(6 \%)$ and apical (1\%) segments. We found no significant difference in the prevalence of LGE between patients with and without CAD (77\% and 70\%, respectively; $p=0.67)$. Compared with patients without focal fibrosis, LGE-positive subjects had more severe AS, as evidenced by smaller AVA index $(p=0.018)$, thicker LV walls $(p<0.001)$ and higher LV mass index $(p=0.009)$. Patients with LGE also had higher levels of BNP $(p=0.004)$ and Hs-Tn-I $(p=0.003)$. The patients' clinical and imaging characteristics stratified by the presence of LGE are summarised in Tables 1 and 2.

\section{Longitudinal deformation analysis}

The mean GLS was $-18 \pm 5 \%$ (range: $-3 \%$ to $-31 \%$ ), and a reduction in GLS > $-20 \%$ was observed in $61 \%$ of patients.

To analyse GLS with regard to clinical and structural parameters, we dichotomised the variables (above and below median: $-18.5 \%$; Table 3). Patients with lower GLS had more severe AS, based on smaller AVA index $(p=0.018)$, higher mean transvalvular gradient $(p=0.004)$, lower systolic blood pressure $(p=0.005)$ and greater QRS voltage on the ECG $(p=0.011)$. The low-GLS group also had thicker LV walls $(p=0.009)$, higher LV volumes $(p<0.001)$, greater LV mass index $(p<0.001)$ and lower LVEF $(p<0.001)$. This group showed signs of elevated LV filling pressures, as evident by higher E/e' ratios $(p=0.011)$, with consequently higher LA volume index $(p=0.002)$ and pulmonary artery systolic pressure $(p=0.031)$. Higher levels of BNP $(p=0.001)$ and Hs-Tn-I $(p=0.002)$ were detected in these patients. Representative images of patients with various degrees of LV remodelling by echocardiography, CMR and histology are shown in Fig. 4.

\section{Analysis of associations}

CVF correlated with LV end-diastolic diameter $(\mathrm{r}=0.242$, $p=0.043), \mathrm{LV}$ end-systolic volume $(\mathrm{r}=0.265, p=0.028)$, LVEF $(\mathrm{r}=-0.246, p=0.04)$ and LA volume index $(\mathrm{r}=0.314, p=0.009)$. When subendocardium was excluded from the analysis, CVF correlated with LV mass $(\mathrm{r}=0.247, p=0.041), \operatorname{LVEF}(\mathrm{r}=-0.354, p=0.003)$, GLS $(\mathrm{r}=-0.303, p=0.013)$ and BNP $(\mathrm{r}=0.242, p=0.045)$ (Fig. 5). Native T1, ECV and indexed ECV did not associate with CVF.

With regards to LV structure and function, GLS correlated with $\mathrm{LV}$ end-diastolic volume $(\mathrm{r}=-0.485, p<0.001)$, $\mathrm{LV}$ end-systolic volume $(\mathrm{r}=-0.636, p<0.001), \mathrm{LV}$ mass index $(\mathrm{r}=-0.615, p<0.001)$ and LVEF $(\mathrm{r}=0.7, p<001)$. GLS was also linked to parameters that were associated with elevated LV filling pressures: mean $\mathrm{E} / \mathrm{e}^{\prime}(\mathrm{r}=-0.4$, $p=0.002)$, LA volume index $(\mathrm{r}=-0.405, p<0.001)$ and estimated pulmonary artery systolic pressure $(\mathrm{r}=-0.376$, $p<0.05)$. Native T1 correlated with LV end-systolic volume $(\mathrm{r}=0.349, p=0.003), \mathrm{LV}$ end-diastolic volume $(\mathrm{r}=0.269, p=0.03)$, LV mass index $(\mathrm{r}=0.414, p<0.001)$ and LVEF $(r=0.317, p<0.05)$. GLS and native T1 were associated with the degree of AS severity: AV mean gradient $(\mathrm{r}=-0.387, p<0.001$ and $\mathrm{r}=0.408, p<0.001$, respectively) and AVA ( $\mathrm{r}=0.30, p<0.05$ and $\mathrm{r}=0.3, p=0.02$, respectively).

With regard to serum biomarkers, GLS and native T1 correlated with BNP $(r=-0.653, p<0.001$ and $\mathrm{r}=0.371$, $\mathrm{p}<0.05$, respectively) and hs-Tn-I $(\mathrm{r}=-0.486, p<0.001$ 
Table 2 Cardiovascular imaging and histology data of study cohort, stratified by the presence of focal fibrosis

\begin{tabular}{|c|c|c|c|c|}
\hline Echocardiography data $(n=83)$ & All patients $(n=83)$ & LGE $(+)$ patients $(n=61)$ & LGE (-) patients $(n=22)$ & $P$-value \\
\hline Peak AV velocity, $\mathrm{m} / \mathrm{s}$ & $4.8 \pm 0.6$ & $4.9 \pm 0.6$ & $4.6 \pm 0.5$ & 0.074 \\
\hline Mean AV gradient, $\mathrm{mm} \mathrm{Hg}$ & $57.8 \pm 16$ & $59.8 \pm 17$ & $52.4 \pm 14$ & 0.071 \\
\hline Low gradient AS & $10(12 \%)$ & $6(10 \%)$ & $4(18 \%)$ & 0.422 \\
\hline AVA, $\mathrm{cm}^{2}$ & $0.84 \pm 0.2$ & $0.83 \pm 0.2$ & $0.88 \pm 0.2$ & 0.364 \\
\hline AVA index, $\mathrm{cm}^{2} / \mathrm{m}^{2}$ & $0.44 \pm 0.1$ & $0.43 \pm 0.1$ & $0.49 \pm 0.1$ & 0.018 \\
\hline IVSd, mm & $12.7 \pm 1.7$ & $13.1 \pm 1.5$ & $11.5 \pm 1.5$ & $<0.001$ \\
\hline Posterior wall diameter, $\mathrm{mm}$ & $11.5 \pm 1.4$ & $11.9 \pm 1.3$ & $10.3 \pm 1.2$ & $<0.001$ \\
\hline LVdd, mm & $51.4 \pm 5.4$ & $52.1 \pm 5.4$ & $49.3 \pm 4.9$ & 0.034 \\
\hline LVsd, mm & $32.7 \pm 5.9$ & $33.1 \pm 6.1$ & $31.7 \pm 5.6$ & 0.362 \\
\hline $\mathrm{E} / \mathrm{A}$ & $1.1 \pm 0.5$ & $1.1 \pm 0.5$ & $1.2 \pm 0.3$ & 0.615 \\
\hline E deceleration time, $\mathrm{ms}$ & $259 \pm 70$ & $257 \pm 69$ & $262 \pm 74$ & 0.813 \\
\hline E/e' septal & $17.6 \pm 7$ & $17.9 \pm 6.3$ & $16.8 \pm 9.5$ & 0.619 \\
\hline E/e' lateral & $14.5 \pm 6$ & $15 \pm 6.5$ & $13.2 \pm 5.8$ & 0.276 \\
\hline E/e' mean & $15.6 \pm 6$ & $16 \pm 5.9$ & $14.3 \pm 5.7$ & 0.254 \\
\hline LA volume index, $\mathrm{ml} / \mathrm{m}^{2}$ & $47.9 \pm 12$ & $49.2 \pm 12$ & $44.7 \pm 12$ & 0.129 \\
\hline PASP, mm Hg & $38 \pm 15$ & $40.5 \pm 15$ & $33.6 \pm 12$ & 0.175 \\
\hline $\mathrm{RV} \mathrm{S}^{\prime}, \mathrm{cm} / \mathrm{s}$ & $11.6 \pm 3$ & $11.4 \pm 3$ & $12 \pm 2$ & 0.377 \\
\hline TAPSE & $21.7 \pm 3$ & $21.7 \pm 4$ & $21.8 \pm 3$ & 0.924 \\
\hline GLS, $\% *$ & $-18 \pm 5$ & $-17.5 \pm 4.8$ & $-19.4 \pm 5.3$ & 0.147 \\
\hline \multicolumn{5}{|l|}{$C M R$ data $(n=83)$} \\
\hline IVSd, mm & $13.3 \pm 2$ & $13.6 \pm 2$ & $12.6 \pm 2$ & 0.062 \\
\hline LVdd, mm & $50.6 \pm 6$ & $51 \pm 6$ & $49.3 \pm 6$ & 0.264 \\
\hline LVsd, mm & $33.8 \pm 8$ & $34.2 \pm 8$ & $33 \pm 9$ & 0.561 \\
\hline LVEDV, ml & $144.3 \pm 44$ & $149.7 \pm 44$ & $130 \pm 44$ & 0.079 \\
\hline LVESV, ml & $51(28-61)$ & $46(31-69)$ & $29(24-45)$ & 0.106 \\
\hline LV stroke volume index, $\mathrm{ml} / \mathrm{m}^{2}$ & $48 \pm 11$ & $48.3 \pm 10$ & $48.4 \pm 11$ & 0.982 \\
\hline LVEF, \% & $66.8 \pm 13$ & $65.3 \pm 13$ & $70.8 \pm 12$ & 0.088 \\
\hline $\mathrm{LVEF}<50 \%$ & $9(11 \%)$ & $8(13 \%)$ & $1(5 \%)$ & 0.427 \\
\hline LV mass index, $\mathrm{g} / \mathrm{m}^{2}$ & $97.6 \pm 32$ & $103.4 \pm 32$ & $82.6 \pm 29$ & 0.009 \\
\hline RVEDV, ml & $125.3 \pm 31$ & $129.5 \pm 31$ & $114.2 \pm 31$ & 0.052 \\
\hline RVESV, ml & $49.3 \pm 18$ & $49.7 \pm 19$ & $48.3 \pm 17$ & 0.747 \\
\hline RVEF, \% & $60.8 \pm 10$ & $61.9 \pm 10$ & $58 \pm 8$ & 0.111 \\
\hline Native $\mathrm{T} 1, \mathrm{~ms}^{\#}$ & $959.7 \pm 34$ & $961.8 \pm 31$ & $952.5 \pm 43$ & 0.359 \\
\hline Post-contrast $\mathrm{T} 1, \mathrm{~ms}^{\#}$ & $351(326-362)$ & $361(325-376)$ & $350(326-358)$ & 0.415 \\
\hline $\mathrm{ECV}, \%^{\#}$ & $22.7 \pm 3.6$ & $23.4 \pm 3.7$ & $22.2 \pm 3.5$ & 0.541 \\
\hline ECV index, $\% / \mathrm{m}^{2}$ & $11.8 \pm 2$ & $12.3 \pm 2$ & $11.3 \pm 2$ & 0.271 \\
\hline \multicolumn{5}{|l|}{ Histology data $(n=71)$} \\
\hline $\mathrm{CVF}, \% \&$ & $15.1(9-21)$ & $15.9(9-19)$ & $12.4(9-24)$ & 0.887 \\
\hline CVF subendocardial, \%\& & $21.1(12-29)$ & $28.7 \%(19-33)$ & $20.7 \%(15-30)$ & 0.040 \\
\hline
\end{tabular}

The boldface values indicate statistical significance

Continuous variables are presented as mean $\pm \mathrm{SD}$ or median [interquartile range]. Categorical variables are expressed as $n(\%)$

$A V$ Aortic valve, $A V A$ Aortic valve area, $E$ Peak early velocity of the transmitral flow, $C M R$ Cardiovascular magnetic resonance, $C V F$ Collagen volume fraction, $e$ ' Peak early diastolic velocity of the mitral annulus displacement, $G L S$ Global longitudinal strain, $E C V$ Extracellular volume, IVSd Interventricular septum diastolic diameter, $L V E D V$ Left ventricular end-diastolic volume, LVESV Left ventricular end-systolic volume, $L V E F$ Left ventricular ejection fraction, $L A$ Left atrium, $L G E$ Late gadolinium enhancement, $L G E(+)$ Patients with late gadolinium enhancement, $L G E(-)$ Patients without late gadolinium enhancement, PASP Pulmonary artery systolic pressure measured by echocardiography, $R V E D V$ Right ventricular end-diastolic volume, $R V E F$ Right ventricular ejection fraction, $R V E S V$ Right ventricular end-systolic volume, $R V S$ ' Peak systolic velocity of the tricuspid annulus displacement, TAPSE Tricuspid annulus plane systolic excursion, *- value based on the data analysis in 77 patients; \#- values based on the data analysis in 67 patients; \&- values based on the data analysis in 71 patient 


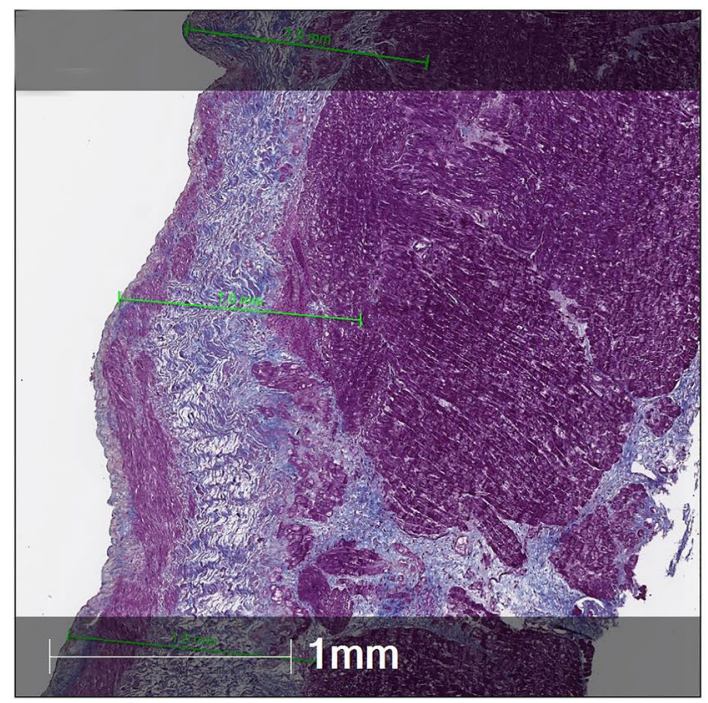

Fig. 3 Image on the left shows myocardial biopsy sample stained with Masson 's trichrome. Graph on the right shows comparison of collagen volume fraction (CVF) in different layers of myocardium.

and $r=0.333, p<0.05$, respectively) and with each other $(\mathrm{r}=-0.321, p<0.05)$ (Fig. 6).

\section{Reproducibility of measurements}

The intraclass correlation coefficients for native $\mathrm{T} 1$ were 0.945 (95\% CI $0.88-0.97$, bias $3.3 \pm 11.0 \mathrm{~ms}$ ) for intraobserver variation and 0.958 (95\% CI 0.91-0.98, bias $9.1 \pm 15.1 \mathrm{~ms}$ ) for inter-observer variation. The GLS measurements also demonstrated excellent reproducibility: 0.969 (95\% CI 0.93-0.98, bias $0.51 \pm 1.3$ ) for intra-observer variation and 0.981 (95\% CI 0.96-0.99, bias $1.5 \pm 1)$ for interobserver variation.

\section{Discussion}

This prospective study presents a comprehensive assessment of the consequences of AS on LV myocardium by integrating CMR and STE data with a large number of myocardial biopsies.

The main study findings are as follows:

(1) The non-infarct type of focal fibrosis is highly prevalent in severe low-risk AS patients and determines more advanced LV remodeling.

(2) Histologically measured myocardial fibrosis is associated with imaging and serum biomarkers of LV systolic dysfunction and left side chamber enlargement.
Higher proportion of collagen detected in subendocardium compared to midmyocardium

(3) The subendocardium is affected by myocardial fibrosis to a greater extent and determines longitudinal dysfunction.

(4) GLS is associated with invasively and non-invasively measured myocardial fibrosis; low GLS and elevated native $\mathrm{T} 1$ differentiated patients with more advanced LV remodelling.

Compared with previous studies in severe AS patients, our cohort was younger and free from significant CAD, thus representing low-risk isolated AS patients. Although $90 \%$ of our study population had preserved LVEF, a more detailed assessment of myocardial structure and function through cardiac imaging and histological analysis revealed evidence of varying degrees of myocardial injury.

The amount of fibrosis in the myocardial biopsies varied substantially, from $2 \%$ to $41 \%$. Diffuse fibrosis, which is present in healthy myocardium, constituted less than $2 \%$, based on the autopsy results of subjects who died of noncardiovascular causes [28, 29]. If the amount of myocardial fibrosis increases with age is less clear. We found that histological myocardial fibrosis was associated with LV and LA enlargement and worse systolic function, underscoring the role of myocardial fibrosis in the pathophysiological progression to cardiac decompensation in AS, in terms morphology and function. Consistent with earlier studies, we found that the subendocardial layer contained more fibrosis compared with a midmyocardium. Gradients of myocardial fibrosis in the LV wall have been described in patients with severe AS and those with hypertrophic cardiomyopathy and hypertensive heart disease-conditions that are both 
Table 3 Patients clinical and imaging characteristics stratified by median GLS and native T1 values

\begin{tabular}{|c|c|c|c|c|c|c|}
\hline & $\mathrm{GLS} \geq-18.5 \%(n=40)$ & GLS $<-18.5 \%(n=37)$ & $P$-value & $\begin{array}{l}\text { Native } \\
\mathrm{T} 1 \geq 957 \mathrm{~ms} \\
(\mathrm{n}=34)\end{array}$ & $\begin{array}{l}\text { Native } \\
\mathrm{T} 1<957 \mathrm{~ms} \\
(\mathrm{n}=33)\end{array}$ & $P$-value \\
\hline Age, yrs & $66 \pm 8$ & $68 \pm 8$ & 0.256 & $65.8 \pm 9$ & $66 \pm 9$ & 0.917 \\
\hline Male gender & $18(45 \%)$ & $14(38 \%)$ & 0.548 & $15(44 \%)$ & $11(33 \%)$ & 0.446 \\
\hline BSA, $m^{2}$ & $1.98 \pm 0.2$ & $1.86 \pm 0.2$ & 0.004 & $1.96 \pm 0.16$ & $1.93 \pm 0.19$ & 0.607 \\
\hline Systolic BP, mmHg & $143 \pm 23$ & $158 \pm 23$ & 0.005 & $139 \pm 21$ & $156 \pm 26$ & 0.006 \\
\hline Diastolic BP, mmHg & $83 \pm 11$ & $85 \pm 11$ & 0.485 & $82 \pm 10$ & $86 \pm 13$ & 0.203 \\
\hline Unobstructive CAD & $20(50 \%)$ & $18(49 \%)$ & 1.0 & $20(59 \%)$ & $14(42 \%)$ & 0.893 \\
\hline Hypertension & $36(90 \%)$ & $33(89 \%)$ & 0.447 & $27(79 \%)$ & $33(100 \%)$ & 0.109 \\
\hline Diabetes mellitus & $8(20 \%)$ & $4(11 \%)$ & 0.768 & $6(18 \%)$ & $7(21 \%)$ & 1.0 \\
\hline NYHA f.cl. $\geq 3$ & $26(65 \%)$ & $14(38 \%)$ & 0.085 & $16(47 \%)$ & $15(46 \%)$ & 0.749 \\
\hline MLHFQ score & $37 \pm 20$ & $32 \pm 20$ & 0.257 & $37 \pm 21$ & $36 \pm 20$ & 0.839 \\
\hline $6 \mathrm{MWT}, \mathrm{m}$ & $351 \pm 105$ & $358 \pm 104$ & 0.767 & $367 \pm 106$ & $352 \pm 94$ & 0.558 \\
\hline \multicolumn{7}{|l|}{$E C G$} \\
\hline $\mathrm{HR}, \mathrm{b} / \mathrm{min}$ & $80 \pm 14$ & $75 \pm 11$ & 0.100 & $78 \pm 4$ & $77 \pm 12$ & 0.742 \\
\hline QRS, ms & $95(90-102)$ & $90(86-98)$ & 0.105 & $94(89-102)$ & $90(84-101)$ & 0.313 \\
\hline $\mathrm{S}-\mathrm{L}, \mathrm{mm}$ & $34 \pm 11$ & $28 \pm 8.5$ & 0.011 & $34 \pm 10$ & $29 \pm 9$ & 0.036 \\
\hline \multicolumn{7}{|l|}{ Echo data } \\
\hline AVA index, $\mathrm{cm}^{2} / \mathrm{m}^{2}$ & $0.42 \pm 0.1$ & $0.47 \pm 0.08$ & 0.018 & $0.4 \pm 0.1$ & $0.45 \pm 0.1$ & 0.075 \\
\hline Peak AV velocity, m/s & $5.0 \pm 0.7$ & $4.7 \pm 0.5$ & 0.055 & $5.0 \pm 0.6$ & $4.8 \pm 0.6$ & 0.105 \\
\hline Mean gradient, $\mathrm{mmHg}$ & $63 \pm 17.7$ & $53 \pm 13.2$ & 0.004 & $64 \pm 16$ & $57 \pm 15$ & 0.052 \\
\hline IVSd, mm & $13.3 \pm 1.8$ & $12.2 \pm 1.4$ & 0.009 & $13 \pm 1.9$ & $12.6 \pm 1.6$ & 0.368 \\
\hline LVdd, mm & $53.7 \pm 12$ & $48.8 \pm 4.7$ & 0.002 & $53 \pm 5$ & $50 \pm 5$ & 0.049 \\
\hline LVsd, mm & $35.4 \pm 6$ & $29.6 \pm 4$ & $<0.001$ & $35 \pm 6$ & $32 \pm 6$ & 0.057 \\
\hline E deceleration time, ms & $254 \pm 76$ & $264 \pm 67$ & 0.542 & $252 \pm 68$ & $266 \pm 75$ & 0.759 \\
\hline E/e' septal & $17.1(14-22)$ & $14(11.7-18)$ & 0.011 & $16.5(12.8-18)$ & $16(12-20)$ & 0.845 \\
\hline E/e' mean & $17.4 \pm 6.9$ & $14.2 \pm 4.4$ & 0.021 & $15 \pm 5$ & $16 \pm 7$ & 0.909 \\
\hline LA volume index, $\mathrm{ml} / \mathrm{m}^{2}$ & $53 \pm 12$ & $44 \pm 11$ & 0.002 & $48 \pm 9$ & $48 \pm 15$ & 0.473 \\
\hline PASP, mmHg & $43.5 \pm 18$ & $32.9 \pm 7$ & 0.031 & $41 \pm 17$ & $37 \pm 12$ & 0.947 \\
\hline GLS, $\%$ & $14.3 \pm 3.9$ & $21.7 \pm 2.7$ & $<0.001$ & $16.7 \pm 5.6$ & $18.2 \pm 4$ & 0.120 \\
\hline GLS $>-15 \%$ & $16(40 \%)$ & - & $<0.001$ & $10(29 \%)$ & $4(12 \%)$ & 0.049 \\
\hline \multicolumn{7}{|l|}{ CMR and histology data } \\
\hline IVSd, mm & $14 \pm 2$ & $12.6 \pm 2$ & 0.005 & $14 \pm 1.6$ & $13 \pm 2.3$ & 0.364 \\
\hline LVdd, mm & $53 \pm 7$ & $48.3 \pm 5$ & $<0.001$ & $52 \pm 6$ & $50 \pm 5$ & 0.074 \\
\hline LVsd, mm & $37 \pm 9$ & $30.6 \pm 6$ & $<0.001$ & $36.5 \pm 7$ & $32 \pm 6$ & 0.009 \\
\hline LVEDV, ml & $160.7 \pm 48$ & $126 \pm 35$ & $<0.001$ & $153 \pm 40$ & $143 \pm 44$ & 0.201 \\
\hline LVESV, ml & $56.9(41-77)$ & $29(24-41)$ & $<0.001$ & $52(37-72)$ & $41(28-53)$ & 0.083 \\
\hline LVEF, \% & $59 \pm 14$ & $74 \pm 7$ & $<0.001$ & $62.4 \pm 14$ & $68 \pm 12$ & 0.053 \\
\hline LVEF $<50 \%$ & $8(20 \%)$ & 0 & 0.009 & $6(18 \%)$ & $2(6 \%)$ & 0.541 \\
\hline LV mass index, $\mathrm{g} / \mathrm{m}^{2}$ & $113 \pm 33$ & $80.6 \pm 24$ & $<0.001$ & $109 \pm 31$ & $91 \pm 30$ & 0.021 \\
\hline LGE prevalence & $34(85 \%)$ & $23(62 \%)$ & 0.058 & $27(79 \%)$ & $25(76 \%)$ & 0.802 \\
\hline Native T1, ms & $967 \pm 31$ & $950 \pm 37$ & 0.066 & $987 \pm 26$ & $936 \pm 18$ & $<0.001$ \\
\hline Post-contrast T1, ms & $349(326-354)$ & $355(332-366)$ & 0.201 & $352(328-362)$ & $348(318-362)$ & 0.445 \\
\hline $\mathrm{ECV}, \%$ & $22.3 \pm 4$ & $22.9 \pm 2.4$ & 0.456 & $23 \pm 3.2$ & $22 \pm 3.9$ & 0.243 \\
\hline $\mathrm{T} 2, \mathrm{~ms}$ & $43(41-45)$ & $42(40-44)$ & 0.196 & $43.3(41-45)$ & $42(40-44)$ & 0.291 \\
\hline BNP, pg/l & $252(98-813)$ & $79(59-173)$ & 0.001 & $163(73-581)$ & $120(62-260)$ & 0.413 \\
\hline Hs-Tn-I, pg/l & $15(7.5-29)$ & $6.9(5-12.9)$ & 0.002 & $14(7-27)$ & $7.5(5-16)$ & 0.089 \\
\hline CVF, \% & $17.2(10-22)$ & $13.5(8-20)$ & 0.279 & $18.1(8-24)$ & $13.4(10-21)$ & 0.564 \\
\hline CVF subendocardial, $\%$ & $23.4(13-33)$ & $18.4(11-27)$ & 0.199 & $22.3(9-28)$ & $18.8(12-26)$ & 0.855 \\
\hline
\end{tabular}

Continuous variables are presented as mean $\pm \mathrm{SD}$ or median [interquartile range]. Categorical variables are expressed as $\mathrm{n}(\%)$. The boldface values indicate statistical significance. Abbreviations as in Tables 1 and 2 


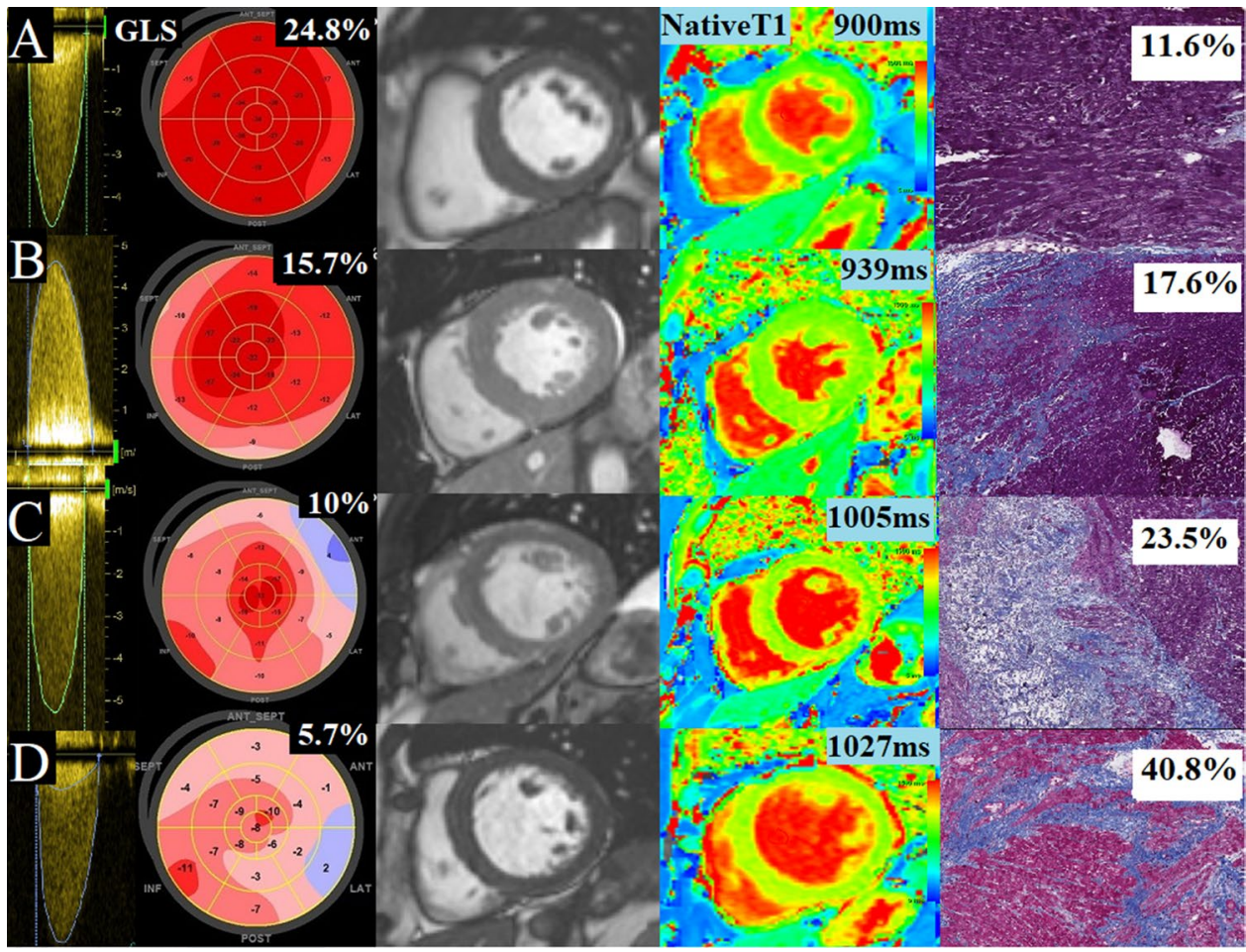

Fig. 4 Four exemplar patients showing progressive cardiac remodeling: continuous-wave Doppler (maximum velocities $>4 \mathrm{~m} / \mathrm{s}$; Column 1), global longitudinal strain (GLS; Column 2), short axis cine stills demonstrating degrees of left ventricular (LV) remodeling (Column 3), matching native T1 (Column 4) and collagen volume fraction (CVF) in myocardial biopsies stained with Masson 's trichrome (Column 5). Patient A has preserved GLS, minimal LV hypertrophy, low native T1 and CVF of 11.6\%. Patient B has reduced GLS, concentric LV hypertrophy, higher native $\mathrm{T} 1$ and moderate histological fibrosis (CVF-17.6\%). Patient C has low GLS, evidence of LV hypertrophy, high native $\mathrm{T} 1$ and significant histological fibrosis (CVF-23.5\%). Patient $\mathrm{D}$, with decompensated heart failure, has low GLS, LV cavity dilatation, high native $\mathrm{T} 1$ and extensive histological fibrosis (CVF$40.8 \%$ )

represented by LVEF, because it can be compensated by global radial function [7, 31].

Notably, patient groups did not differ by symptom status, functional capacity or quality of life assessment. This finding suggests that symptom assessments can be challenging and misleading and do not always reflect true cardiac condition, indicating that the decision to intervene should be supported by objective markers of cardiac injury, rather than based on subjective assessment of symptom status.

Imaging biomarkers, or the integration of several parameters, might be particularly useful in patients with no or minimal symptoms or when ascertaining valve-related symptoms is challenging. Our data implicate GLS and native T1 as early markers of cardiac decompensation. GLS can also be used as a surrogate marker of myocardial fibrosis, as it was associated with both, invasively and non-invasively measured myocardial fibrosis.

Seventy-four percent of our patients had areas of focal fibrosis, $98 \%$ of which were the non-infarct type and which were independent of the presence of nonobstructive CAD. Although only 1 or 2 segments were affected by LGE in most patients, data from a recent large multicentre study show that $>2 \%$ of 

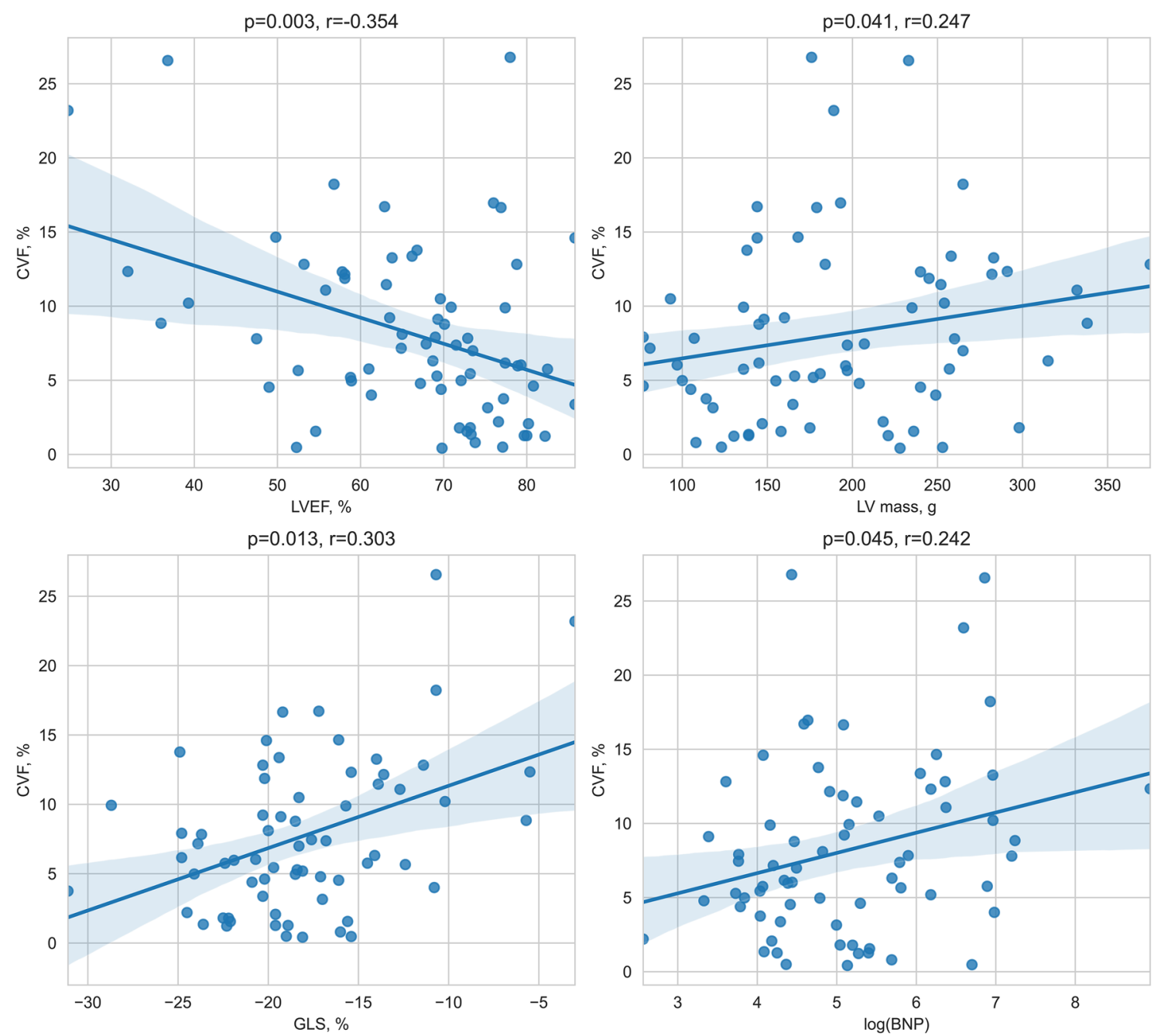

Fig. 5 Correlations between histological myocardial fibrosis (CVF) and LV ejection fraction (a), LV mass (b), GLS (c) and brain natriuretic peptide (BNP) (d) are shown. Abbrevations are as in Fig. 4
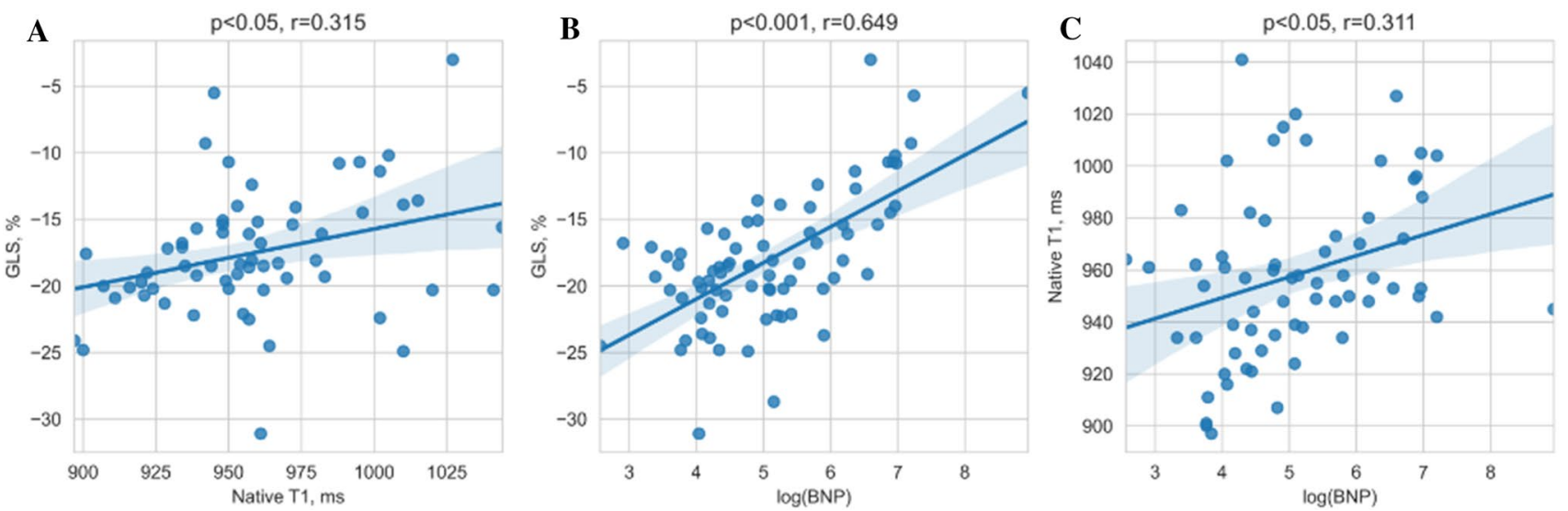

Fig. 6 Correlations between GLS and native T1 (a), GLS and BNP (b), native T1 and BNP (c) are shown. Abbrevations are as in Figs. 4 and 5 
LGE in patients with severe AS who undergo AVR is associated with worse postoperative survival [32]. We found, that the myocardium of patients who have progressed to more advanced myocardial injury and have developed areas of irreversible replacement fibrosis on CMR also contains higher degree of diffuse fibrosis measured histologically. Unexpectedly, we found no associations between CVF and CMR markers of diffuse fibrosis, for which there are several explanations. There was a possible sampling error, because only 1 biopsy sample per patient was analysed. Further, the histological and T1 mapping analyses were performed at different levels and layers of the interventricular septum. The myocardial biopsies were endocardial and taken from the basal anteroseptum, possibly containing higher amounts of fibrotic tissue, and the region of interest for the T1 mapping measurements was drawn in the middle of the septum at the midventricular level, avoiding endo and epicardial borders. The data in this field are inconsistent, with some studies reporting significant associations between invasively and non-invasively measured myocardial fibrosis in AS cohorts [15, 33] and others failing to demonstrate this association $[24,29]$.

Although our patients presented with increased LV mass and myocardial fibrosis in the histological analysis, ECV values were not elevated in our cohort compared with our local reference range. This finding can be explained by the greater increase in cellular mass (adaptive hypertrophy), as opposed to the expansion of extracellular space, because ECV per se represents the percentage of space that is occupied by the extracellular compartment of the total LV mass. The average native T1 and ECV values in our cohort were lower in comparison to AS populations in other studies [13, 15]. A large T1 mapping data variability across different centers have been previously reported, influenced by differences in field strength, vendor-specific set-up and variations in sequences $[34,35]$. Disparities in ECV values can also be expected with the non-uniformity of contrast agents and their doses [36]. Another explanation for such variability relates to differences in the study cohorts. When interpreting our results, we should consider that we examined relatively young, low-risk patients who were free from significant CAD, whereas other studies, especially those that included transcatheter treatment cohorts, enrolled patients who were in their $80 \mathrm{~s}$ and had a higher rate of comorbidities [37].

\section{Study limitations}

The study was composed of a small number of AS patients, however it included substantial number of myocardial biopsies. Due to the Covid-19 pandemic, delays in patient examinations and surgeries were experienced, causing uneven time frames between the preoperative patient assessment (echocardiographic and CMR) and surgery with myocardial sampling, potentially affecting the final result. Proportion of histologically measured myocardial fibrosis could have been affected by the size and depth of biopsy samples, as more superficially sampled and smaller biopsies may contain higher proportion of fibrotic tissue in comparison to larger biopsy samples. Although measuring T1 values only in the septum is a validated and common method, it might not represent the entire myocardium. Because we excluded patients with comorbidities, such as obstructive CAD, a history of myocardial infarction, renal failure and persistent atrial arrhythmias, our results should not be overgeneralized to the broader AS patient population. Another limitation of our study is that the increase in type I error across the statistical analyses was not controlled.

\section{Conclusion}

A comprehensive assessment of LV response to AS by integrating histology, CMR and STE reveals varying degrees of myocardial injury that are not apparent with traditional measures of LV systolic function. Histological myocardial fibrosis was associated with imaging and serum biomarkers of LV systolic dysfunction and left side chamber enlargement. We found that native T1 by CMR and GLS by STE differentiated patients with advanced cardiac remodelling, constituting a marker of subclinical cardiac damage. Of all imaging parameters, only GLS was associated with invasively and non-invasively measured myocardial fibrosis, demonstrating its potential as a surrogate marker of myocardial fibrosis.

Author contributions SG and PS are chief investigators; they conceived the study, led the proposal and protocol development. GB drafted the manuscript. PŠ, AA and TZ contributed to study implementation. NV and DP performed CMR scanning and data analysis. KR, VJ and AZ performed aortic valve replacement surgeries and sampled myocardial biopsies. EŽ and JB performed histological analysis. VS conceived and developed the statistical aspects of the study. All authors reviewed and approved the final version of the manuscript.

Funding Study is funded by the Research Council of Lithuania under 2014-2020 European Union investments in Lithuania operational program (09.3.3-LMT-K-712). The funder had no role in study design, execution, interpretation of the data, or decision to submit results.

\section{Declarations}

Conflict of interest The authors declare that they have no competing interests.

Ethical approval The study conformed to the principles of the Helsinki Declaration, and all subjects gave written consent to participate. The study (protocol, including qualitative and quantitative aspects, and trial materials, including patient information and consent form) was reviewed and approved by the Biomedical Research Ethics Committee of the Vilnius Region (16/March/2018; No: 158200-18/9-1014-558). 
Consent to participate Informed consent was obtained from all individual participants included in the study.

Consent to publish The authors affirm that human research participants provided informed consent for publication of the images in Fig. 4.

\section{References}

1. Unverferth DV, Baker PB, Swift SE, Chaffee R, Fetters JK, Uretsky BF et al (1986) Extent of myocardial fibrosis and cellular hypertrophy in dilated cardiomyopathy. Am J of Cardiol 57(10):816-820. https://doi.org/10.1016/0002-9149(86)90620-x

2. Anderson KR, Sutton MG, Lie JT (1979) Histopathological types of cardiac fibrosis in myocardial disease. J Pathol 128(2):79-85. https://doi.org/10.1002/path.1711280205

3. Chin CWL, Everett RJ, Kwiecinski J, Vesey AT, Yeung E, Esson $\mathrm{G}$ et al (2017) Myocardial fibrosis and cardiac decompensation in aortic stenosis. JACC Cardiovasc Imaging 10:1320-1333

4. Dweck MR, Joshi S, Murigu T, Alpendurada F, Jabbour A, Melina $\mathrm{G}$ et al (2011) Midwall fibrosis is an independent predictor of mortality in patients with aortic stenosis. J Am Coll Cardiol 58:1271-1279

5. Villari B, Campbell SE, Hess OM, Mall G, Vassalli G, Weber KT et al (1993) Influence of collagen network on left ventricular systolic and diastolic function in aortic valve disease. J Am Coll Cardiol 22:1477-1484

6. Barone-Rochette G, Pierard S, Meester De, de Ravenstein C, Seldrum S, Melchior J, Maes F et al (2014) Prognostic significance of LGE by CMR in aortic stenosis patients undergoing valve replacement. J Am Coll Cardiol 64:144-154

7. Weidemann F, Herrmann S, Stork S, Niemann M, Frantz S, Lange $\mathrm{V}$ et al (2009) Impact of myocardial fibrosis in patients with symptomatic severe aortic stenosis. Circulation 120:577-584

8. Chin CW, Semple S, Malley T, White AC, Mirsadraee S, Weale PJ et al (2014) Optimization and comparison of myocardial T1 techniques at $3 \mathrm{~T}$ in patients with aortic stenosis. Eur Heart J Cardiovasc Imaging 15:556-565

9. Balciunaite G, Skorniakov V, Rimkus A, Zaremba T, Palionis D, Valeviciene $\mathrm{N}$ et al (2020) Prevalence and prognostic value of late gadolinium enhancement on CMR in aortic stenosis: metaanalysis. Eur Radiol 30(1):640-651

10. Musa TA, Treibel TA, Vassiliou VS, Captur G, Singh A, Chin C et al (2018) Myocardial scar and mortality in severe aortic stenosis. Circulation 138(18):1935-1947. https://doi.org/10.1161/ CIRCULATIONAHA.117.032839

11. Treibel TA, Kozor R, Schofield R, Benedetti G, Fontana M, Bhuva AN et al (2018) Reverse myocardial remodeling following valve replacement in patients with aortic stenosis. J Am Coll Cardiol 71:860-871

12. Everett RJ, Tastet L, Clavel MA, Chin CWL, Capoulade R, Vassiliou VS et al (2018) Progression of hypertrophy and myocardial fibrosis in aortic stenosis: a multicenter cardiac magnetic resonance study. Circ Cardiovasc Imaging 11:e007451

13. Everett RJ, Treibel TA, Fukui M, Lee H, Rigolli M, Singh A et al (2020) Extracellular myocardial volume in patients with aortic stenosis. J Am Coll Cardiol 75(3):304-316. https://doi.org/10. 1016/j.jacc.2019.11.032

14. Chin CW, Pawade TA, Newby DE, Dweck MR (2015) Risk stratification in patients with aortic stenosis using novel imaging approaches. Circ Cardiovasc Imaging 8(8):e003421. https://doi. org/10.1161/CIRCIMAGING.115.003421
15. Park SJ, Cho SW, Kim SM, Ahn J, Carriere K, Jeong DS et al (2019) Assessment of myocardial fibrosis using multimodality imaging in severe aortic stenosis: comparison with histologic fibrosis. JACC Cardiovasc Imaging 12(1):109-119. https://doi. org/10.1016/j.jcmg.2018.05.028

16. Hwang IC, Kim HK, Park JB, Park EA, Lee W, Lee SP et al (2020) Aortic valve replacement-induced changes in native $\mathrm{T} 1$ are related to prognosis in severe aortic stenosis: T1 mapping cardiac magnetic resonance imaging study. Eur Heart J Cardiovasc Imaging 21(6):653-663. https://doi.org/10.1093/ehjci/jez201

17. $\mathrm{Ng}$ ACT, Prihadi EA, Antoni ML, Bertini M, Ewe SH, Ajmone Marsan N et al (2018) Left ventricular global longitudinal strain is predictive of all-cause mortality independent of aortic stenosis severity and ejection fraction. Eur Heart J Cardiovasc Imaging 19:859-867

18. Vahanian A, Beyersdorf F, Praz F, Milojevic M, Baldus S, Bauersachs J et al (2021) ESC/EACTS Scientific Document Group, $2021 \mathrm{ESC} / \mathrm{EACTS}$ Guidelines for the management of valvular heart disease: Developed by the Task Force for the management of valvular heart disease of the European Society of Cardiology (ESC) and the European Association for Cardio-Thoracic Surgery (EACTS). Eur Heart J. https://doi.org/10.1093/eurheartj/ehab395

19. Harris PA, Taylor R, Thielke R, Payne J, Gonzalez N, Conde JG (2009) Research electronic data capture (REDCap)-a metadatadriven methodology and workflow process for providing translational research informatics support. J Biomed Inform 42(2):377381. https://doi.org/10.1016/j.jbi.2008.08.010

20. Lang RM, Badano LP, Mor-Avi V, Afilalo J, Armstrong A, Ernande L et al (2015) Recommendations for cardiac chamber quantification by echocardiography in adults: an update from the American Society of Echocardiography and the European Association of Cardiovascular Imaging. J Am Soc Echocardiogr 28:1-39

21. Baumgartner H, Hung J, Bermejo J, Chambers JB, Edvardsen $\mathrm{T}$, Goldstein S et al (2017) Recommendations on the echocardiographic assessment of aortic valve stenosis: a focused update from the European Association of Cardiovascular Imaging and the American Society of Echocardiography. Eur Heart J Cardiovasc Imaging 8:254-275

22. Voigt JU, Pedrizzetti G, Lysyansky P, Marwick TH, Houle H, Baumann R et al (2015) Definitions for a common standard for 2D speckle tracking echocardiography: consensus document of the EACVI/ASE/Industry Task Force to standardize deformation imaging. Eur Heart J Cardiovasc Imaging 16:1-11

23. Taylor AJ, Salerno M, Dharmakumar R, Jerosch-Herold M (2016) T1 mapping: basic techniques and clinical applications. JACC Cardiovasc Imaging 9:67-81. https://doi.org/10.1016/j.jcmg.2015. 11.005

24. Messroghli DR, Moon JC, Ferreira VM, Grosse-Wortmann L, He T et al (2017) Clinical recommendations for cardiovascular magnetic resonance mapping of $\mathrm{T} 1, \mathrm{~T} 2, \mathrm{~T} 2 *$ and extracellular volume: A consensus statement by the Society for Cardiovascular Magnetic Resonance (SCMR) endorsed by the European Association for Cardiovascular Imaging (EACVI). J Cardiovasc Magn Reson 19:75. https://doi.org/10.1186/s12968-017-0389-8

25. Ugander M, Oki AJ, Hsu LY, Kellman P, Greiser A, Aletras AH et al (2012) Extracellular volume imaging by magnetic resonance imaging provides insights into overt and sub-clinical myocardial pathology. Eur Heart J 33:1268-1278

26. Horai Y, Mizukawa M, Nishina H, Nishikawa S, Ono Y, Takemoto $\mathrm{K}$ et al (2019) Quantification of histopathological findings using a novel image analysis platform. J Toxicol Pathol 32(4):319-327. https://doi.org/10.1293/tox.2019-0022

27. R Core Team. R: A language and environment for statistical computing (2021) R Foundation for Statistical Computing, Vienna, Austria. R version 4.1.2 (2021-11-01) - "Bird Hippie". https:// www.R-project.org/ 
28. Tanaka M, Fujiwara H, Onodera T, Wu DJ, Hamashima Y, Kawai C (1986) Quantitative analysis of myocardial fibrosis in normals, hypertensive hearts, and hypertrophic cardiomyopathy. Br Heart J 55(6):575-81. https://doi.org/10.1136/hrt.55.6.575

29. Treibel TA, López B, González A, Menacho K, Schofield RS, Ravassa S et al (2018) Reappraising myocardial fibrosis in severe aortic stenosis: an invasive and non-invasive study in 133 patients. Eur Heart J 39(8):699-709. https://doi.org/10.1093/eurheartj/ ehx353

30. Galiuto L, Lotrionte M, Crea F, Anselmi A, Biondi-Zoccai GG, De Giorgio F et al (2006) Impaired coronary and myocardial flow in severe aortic stenosis is associated with increased apoptosis: a transthoracic Doppler and myocardial contrast echocardiography study. Heart 92(2):208-12. https://doi.org/10.1136/hrt.2005. 062422

31. Hein S, Arnon E, Kostin S, Schon “burg M, Elsa“sser A, Polyakova $\mathrm{V}$ et al (2003) Progression from compensated hypertrophy to failure in the pressure-overloaded human heart: structural deterioration and compensatory mechanisms. Circulation 107(7):984-91

32. Kwak S, Everett RJ, Treibel TA, Yang S, Hwang D, Ko T et al (2021) Markers of myocardial damage predict mortality in patients with aortic stenosis. J Am Coll Cardiol 78:545-558. https://doi.org/10.1016/j.jacc.2021.05.047

33. Bull S, White SK, Piechnik SK, Flett AS, Ferreira VM, Loudon M et al (2013) Human non-contrast T1 values and correlation with histology in diffuse fibrosis. Heart 99(13):932-7. https://doi.org/ 10.1136/heartjnl-2012-303052
34. Vo HQ, Marwick TH, Negishi K (2020) Pooled summary of native T1 value and extracellular volume with MOLLI variant sequences in normal subjects and patients with cardiovascular disease. Int J Cardiovasc Imaging 36:325-336. https://doi.org/10. 1007/s10554-019-01717-3

35. Kawel N, Nacif M, Zavodni A, Jones J, Liu S, Cibley CT et al (2012) T1 mapping of the myocardium: Intra-individual assessment of the effect of field strength, cardiac cycle and variation by myocardial region. J Cardiovasc Magn Reson 14(1):27. https:// doi.org/10.1186/1532-429X-14-27

36. Dabir D, Child N, Kalra A, Rogers T, Gebker R, Jabbour A et al (2014) Reference values for healthy human myocardium using a T1 mapping methodology: results from the International T1 Multicenter cardiovascular magnetic resonance study. J Cardiovasc Magn Reson 16(1):69. https://doi.org/10.1186/s12968-014-0069-x

37. Puls M, Beuthner BE, Topci R, Vogelgesang A, Bleckmann A, Sitte $M$ et al (2020) Impact of myocardial fibrosis on left ventricular remodelling, recovery, and outcome after transcatheter aortic valve implantation in different haemodynamic subtypes of severe aortic stenosis. Eur Heart J 41(20):1903-1914. https://doi.org/10. 1093/eurheartj/ehaa033

Publisher's Note Springer Nature remains neutral with regard to jurisdictional claims in published maps and institutional affiliations. 